\title{
Effects of Medium-Chain Triacylglycerols (MCT) on the Maillard Reaction

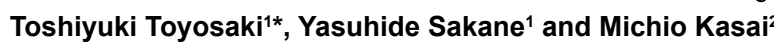

${ }^{1}$ Department of Foods and Nutrition, Koran Women's Junior College Fukuoka, 811-1311, Japan

${ }^{2}$ Central Research Laboratory of The Nisshin OilliO Group LTD 1-Banchi, Shinmei-cho, Yokosuka Kanagawa, 239-0832, Japan

\begin{abstract}
The effects on medium-chain triacylglycerols (MCT) by the Maillard reaction through calcination dough were studied. AGEs generation ratio in the case of 50 min baking dough with MCT has been suppressed advantage compared to other fats. This behavior suggests that the MCT is to suppress the Maillard reaction in the calcination of dough. As a result of measuring a cross-sectional view of the bread cavity holes fired, LCT and MCT showed a downward trend compared to butter and shortening. We have to create a model system to examine whether or not the difference in the impact properties of the solid fat and liquid oil such as Maillard reaction in which the impact. As a result, most of the results were consistent with the results of the actual baking dough was obtained. AGEs produced by Maillard reaction which proceeds during firing has been degraded by the LCT and MCT. On the other hand, butter and shortening degradation of AGEs was suppressed. From this phenomenon, MCT has been presumed that inhibit the formation of AGEs amount as a result of reaction by inhibiting the Maillard reaction.
\end{abstract}

Keywords: Maillard reaction; Medium-Chain Triacylglycerols (MCT); Long-Chain Triacylglycerol (LCT); Advanced Glycation Endproducts (AGEs); Dough; Calcination

\section{Introduction}

Medium-Chain Triacylglycerols (MCT) composed exclusively of medium chain fatty acids (C8 and C10) were first used in the 1950s for dietary treatment of malabsorption syndromes caused by rapid absorption. Since then, they have been widely studied. Although a large number of excellent reports have been published, most of these focus on clinical nutritional or biochemical standpoints [1-9]. In contrast, very few studies have been conducted from a food science standpoint. We previously reported the somewhat interesting finding that, when an MCT is added during the fermentation of bread dough, fermentation of the dough is enhanced. Based on this result, we concluded that MCT are effective for improving bread quality. We also recently found that MCT inhibit the Maillard reaction, which occurs during the baking of bread. The Maillard reaction is a dynamic reaction that occurs during the bread-making process, and is thought to significantly affect the appearance of the baked bread, as well as its flavor and texture. It plays a significant role in the formation of brown color on the surface of bread during baking, and we found that different shades of brown are produced by the Maillard reaction depending on the presence of added lipids, and whether they are solid or liquid. This strongly suggests that the properties of added lipids have some effect on the Maillard reaction, but there have been no reports on these effects. Therefore, we aimed to investigate from various points of view the mechanisms by which MCT are involved in the Maillard reaction during the baking process.

\section{Materials and Methods}

\section{Materials}

Materials were purchased from the following sources. MediumChain Triacylglycerols (MCT) and Long-Chain Triacylglycerols (LCT) were a kind gift from Nisshin OilliO Group Ltd. (Kanagawa, Japan). Spring wheat flour (Super King; $13.8 \%$ protein, $0.42 \%$ ash, $14 \%$ water) was obtained from Nisshin Flour Milling Inc. (Chiyoda, Tokyo, Japan). The contents of protein, ash, lipid and water were $13.1 \%$ (Kjeldahl, $\mathrm{N} \times$ $6.25), 0.4 \%, 1.8 \%$ and $15.0 \%$, respectively. More than $95 \%$ of the flour granules were sifted though the sieve of $132-\mathrm{mm}$ mesh. Dried yeast (Saccharomyces cerevisias) was purchased from S.I. Lesaffre (Marcq-
en-Baroeul, France). Gluten (more than $90 \%$ pure) glucose and lysine were purchased from Nakarai Tesque, Inc. (Kyoto, Japan). Other reagents were of special grade and were obtained from Nacalai Tesque.

\section{Preparation and baking of dough}

Bread dough was prepared using commercially available ingredients for preparing bread dough and by employing the straight dough method. More specifically, dough was prepared using ingredients for preparing a loaf of bread, i.e., lipid, strong flour, live yeast, water, sugar, salt and skimmed milk powder. The lipids used in this experiment were MCT and LCT, and butter and shortening. The added lipid content was $10 \%$. Dough temperature at the completion of mixing was $26^{\circ} \mathrm{C}$, and the dough was fermented for $90 \mathrm{~min}$ at a temperature of 28 to $30^{\circ} \mathrm{C}$. The dough was then molded, and the molded pieces were subjected to final fermentation for 60 to $70 \mathrm{~min}$ at a temperature of $36^{\circ} \mathrm{C}$ and a humidity of $75 \%$, followed by baking for 35 to $40 \mathrm{~min}$ at temperatures of $230^{\circ} \mathrm{C}$ (top of oven) to $210^{\circ} \mathrm{C}$ (bottom of oven). Dough subjected to only primary fermentation 86 was also used in this experiment.

\section{Extraction of advanced glycation endproducts (AGEs), the} Maillard reaction products, from baked bread

AGEs were extracted from $100 \mathrm{~g}$ of baked bread using $50 \mathrm{ml}$ of 0.25 M phosphate buffer ( $\mathrm{pH} 7.4$ ).

\section{Determination of amounts of AGEs}

AGEs are brown pigments, and the absorbance at $420 \mathrm{~nm}$ (brown color development) was thus measured.

*Corresponding author: Toshiyuki Toyosaki, Department of Foods and Nutrition, Koran Women's Junior College, Yokote 1-2-1, Minami-ku, Fukuoka 811-1311, Japan, Tel: +81-92-581-1538; Fax: +81-92-581-2200; E-mail: toyosaki@koran.ac.jp

Received February 05, 2013; Accepted March 15, 2013; Published March 20 2013

Citation: Toyosaki T, Sakane Y, Kasai M (2013) Effects of Medium-Chain Triacylglycerols (MCT) on the Maillard Reaction. J Food Process Technol 4: 224 doi:10.4172/2157-7110.1000224

Copyright: (c) 2013 Toyosaki T, et al. This is an open-access article distributed under the terms of the Creative Commons Attribution License, which permits unrestricted use, distribution, and reproduction in any medium, provided the original author and source are credited. 
Citation: Toyosaki T, Sakane Y, Kasai M (2013) Effects of Medium-Chain Triacylglycerols (MCT) on the Maillard Reaction. J Food Process Technol 4: 224. doi:10.4172/2157-7110.1000224

\section{Preparation of AGEs}

AGEs were prepared in the following manner: $0.5 \mathrm{mM}$ glucose and $0.5 \mathrm{mM}$ lysine were dissolved in $30 \mathrm{ml}$ of distilled water, followed by heating in a water bath for $50 \mathrm{~min}$. The resultant mixture was used as AGEs in the experiment.

Preparation of mixtures of AGEs, gluten and liquid oil or solid fat: In order to investigate the mechanisms of action of MCT on AGEs, we prepared a model system using dough prepared with the same mixing ratio as used for preparing a loaf of bread. After addition of 20 $\mathrm{ml}$ of AGEs prepared in advance, the mixture was mixed for $20 \mathrm{~min}$ before use in the experiment.

\section{Structural analysis using ImageJ}

Cut sections of baked bread were prepared, and images obtained using an optical microscope was loaded onto a PC. Using the "analyze particles" function of ImageJ (Ver. 1.45, free software), changes in pore distribution was measured and analyzed. An example of the analysis ImageJ is shown in Figure 1.

Sodium dodecyl sulfate polyacrylamide gel electrophoresis (SDS-PAGE) of model system sample

The measurement was done according to the method of Laemmli [10], in order to determine the changes with time in the molecular weight of gluten in the model system sample composed of AGEs, gluten and MCT. Electrophoresis was performed using the Mini-Protean II Electrophoresis Cell (Bio-Rad Laboratories, Inc., Japan) at $18 \mathrm{~mA} / \mathrm{gel}$ with Ready Gel J of differing gel concentrations. After electrophoresis, the gels were stained using Coomassive brilliant blue-R250.

\section{Statistical analysis}

All data were presented as mean \pm standard deviation. Statistical comparison between different treatments was done by t-test, which was authorized using Duncan' new multiple range test [11] and application software Stat View 512 only for statistics+(Brain Power, Berkeley, CA, USA). Significance level is $p<0.05$ unless otherwise indicate.

\section{Results and Discussion}

\section{Changes in amount of AGEs produced during baking}

Figure 2 shows the changes in the amount of AGEs produced by the Maillard reaction during baking. When MCT or LCT, which are liquid oils were used, the amount of AGEs tended to be smaller than when butter or shortening, which are solid fats, was used. In particular, when MCT were used, the amount of AGEs was found to be significantly smaller. These results show that liquid oil inhibits the Maillard reaction that occurs during baking when compared with solid fat. Chi-Hao et al. [12] introduced the major AGEs in his recent report on AGEs. In the present study, the structures of the AGEs produced during baking were not determined, and how the different properties of the added lipids, are involved in the Maillard reaction is unknown. Further study is thus needed.

\section{Changes in pore distribution analyzed using ImageJ}

After taking pictures of cut sections of the baked bread, changes in pore distribution were studied using ImageJ (Figure 3). The pore distribution showed a tendency to decrease MCT or LCT, which are liquid oils, were used, as compared with butter or shortening, which are solid fats, was used. Pore distribution significantly affects food texture. These results suggest that using liquid oil results in a less (a)

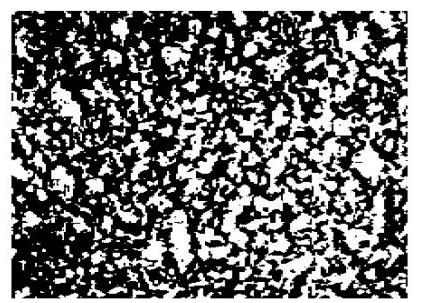

(c)

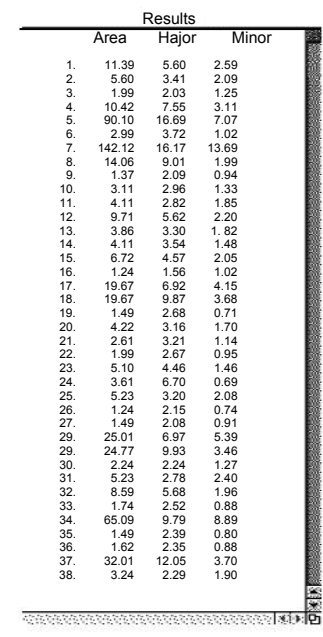

Figure 1: Clarified and emphasized by the cavity hole Threshold function (a) Measurement (b) and the results of the total area of the cavity hole using the function Analyze particle (c)

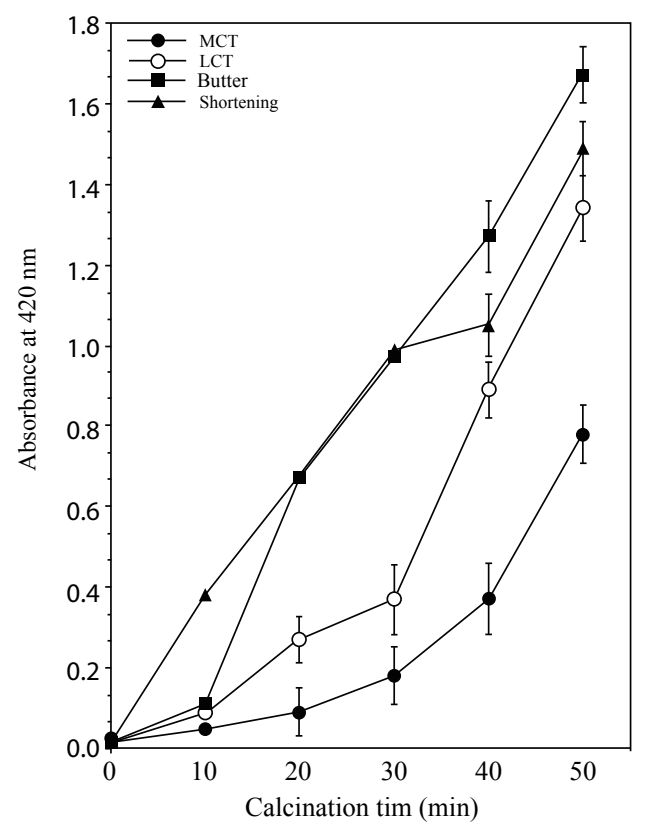

Figure 2: Changes in amount of AGEs produced by the Maillard reaction during baking. 


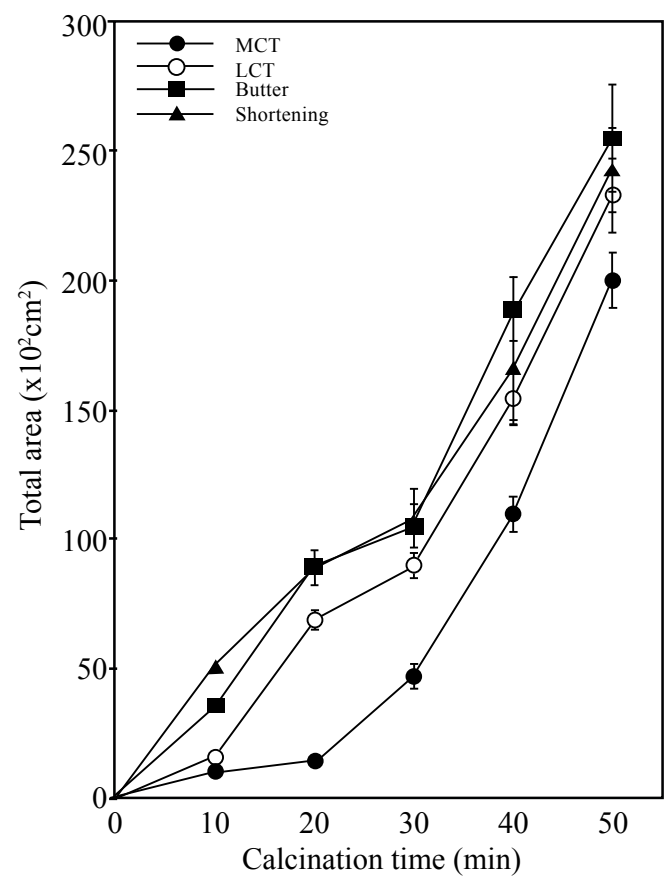

Figure 3: Changes in pore distribution in baked bread.

desirable texture than using solid fat. In order to confirm this, sensory evaluation was performed, and a lower overall evaluation was obtained when liquid oil was used instead of solid fat.

Changes in amount of AGEs produced by the Maillard reaction during baking in model system

The amount of AGEs produced by the Maillard reaction during baking was found to differ significantly depending on whether liquid oil or solid fat was used. When MCT, the amount of AGEs produced by the Maillard reaction was found to decrease. This suggests that MCT themselves inhibit the Maillard reaction was lower than other oils and fats (Figure 4). To clarify how MCT are involved in the Maillard reaction, we prepared a model system. In this experiment, the same results as those shown in figure 2 were obtained, i.e. a larger amount of AGEs was produced when solid fat was used than when liquid oil was used, and the amount of AGEs tended to be less pronounced when MCT were used. These results suggest that MCT are involved in the Maillard reaction in some way, inhibiting the production of AGEs.

Changes in pore distribution in model system analyzed by ImageJ

After obtaining images of cut sections of bread prepared using the model system, changes in the pore distribution were studied using Image J. The results are shown in figure 5. Due to the absence of various additional auxiliary ingredients in the model system, the number of pores tended to increase. When MCT were added, the number of pores was after found to be significantly smaller $(p<0.05)$ than other samples. The quality of the pore distribution depends significantly on the conditions during fermentation, but this study suggests that the quality of the pore distribution is also significantly affected by the Maillard reaction that occurs during baking.

\section{Percentage of added AGEs decomposed during baking}

We investigated the degree of decomposition of added AGEs

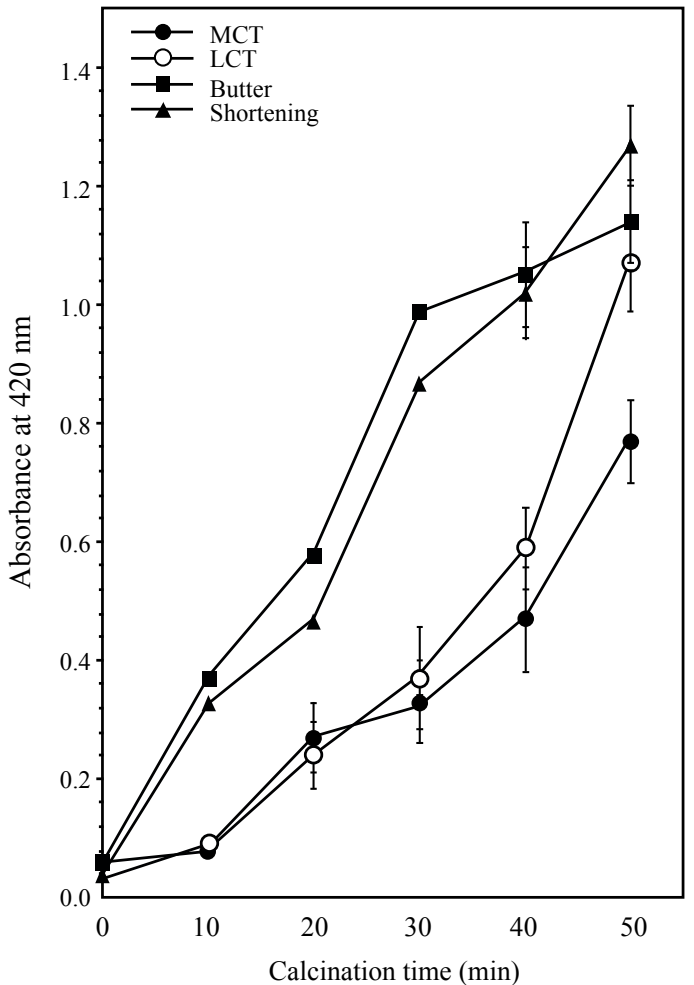

Figure 4: Changes in amount of AGEs produced by the Maillard reaction during baking in the model system.

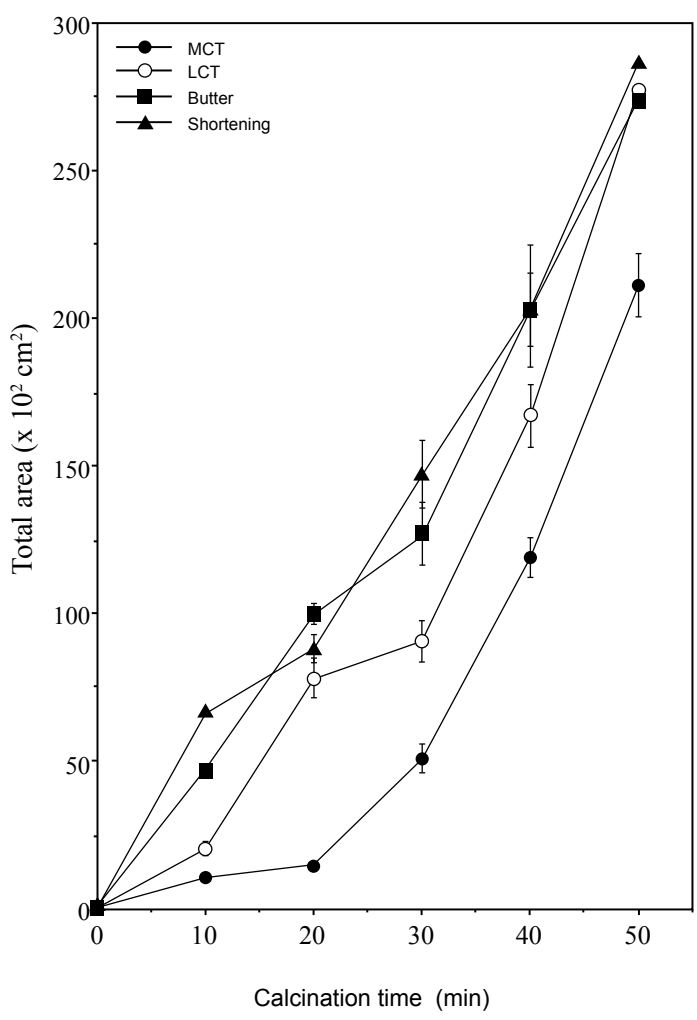

Figure 5: Changes in pore distribution in the model system. 
Citation: Toyosaki T, Sakane Y, Kasai M (2013) Effects of Medium-Chain Triacylglycerols (MCT) on the Maillard Reaction. J Food Process Technol 4: 224. doi:10.4172/2157-7110.1000224

during baking. The results are shown in figure 6. When MCT or LCT was used, the percentage of decomposed AGEs was significantly higher $(p<0.05)$ than solid fats. In contrast, when butter or shortening was used, the percentage of decomposed AGEs was found to be lower. This was due to a difference between solid fat and liquid oil; we believe that the constituent fatty acids play an important role, and this needs to be studied in greater detail in the future.

\section{Changes in molecular weight of gluten in baked bread with or without AGEs}

SDS-PAGE was performed on gluten extracted from the bread baked with or without using liquid oil or solid fat (Figure 7). Bread of AGEs addition, the band A was confirmed in both liquid oil and solid fat. On the other hand, bread of AGEs no addition, the band A could not be confirmed either individual fat and liquid oil. This phenomenon has the potential to change the conformation of the protein was induced by AGEs.

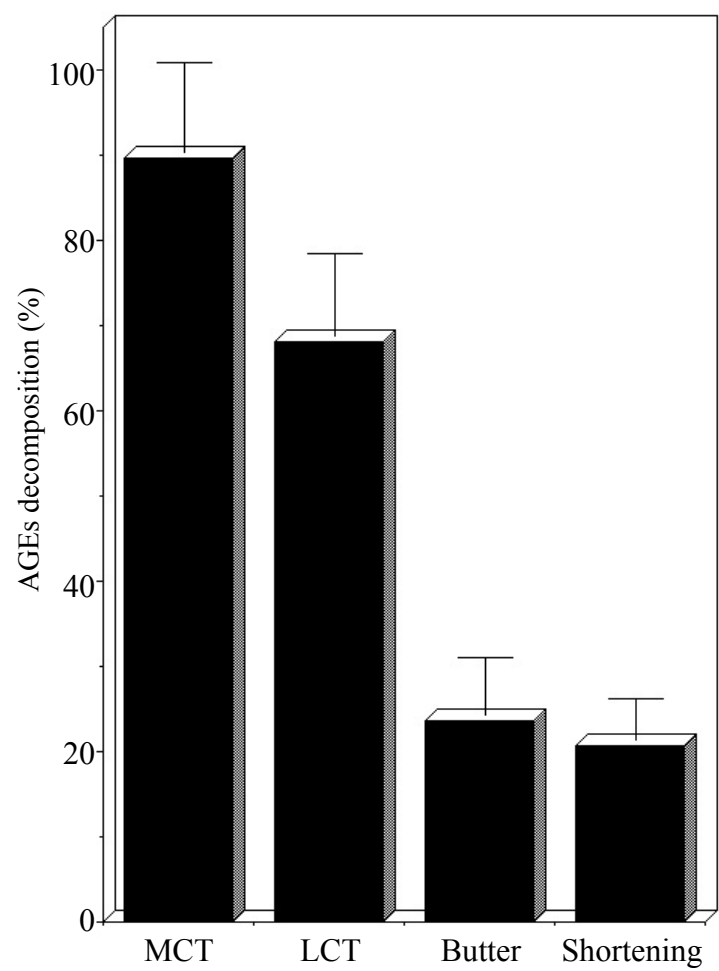

Figure 6: Percentage of added AGEs decomposed during baking.

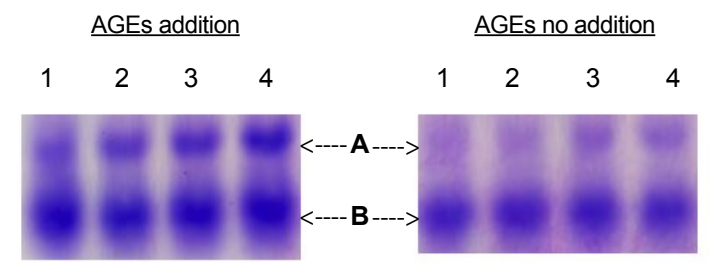

Figure 7: SDS-PAGE of gluten extracted from baked bread. Band A: Heatdenatured gluten, Band B: Unmodified gluten. 1, MCT; 2, LCT; 3, Butter; 4 Shortening.

\section{Decomposition of AGEs and its relationship with MCT}

This study showed that liquid oil at least inhibits AGE production in the Maillard reaction that occurs during baking. In the case of liquid oil (in particular, saturated fatty acids), constituent fatty acids were found to play a significant role in the Maillard reaction, and therefore the relationship with fatty acids needs to be studied in detail in the future.

\section{Conclusion}

AGEs are produced by the Maillard reaction that occurs during 196 the bread baking process. This study revealed that adding liquid oil (in particular MCT) inhibits the amount of AGEs produced, and we believe this finding is novel. We also found that this is caused by the fact that medium chain saturated fatty acids, $\mathrm{C} 8$ and $\mathrm{C} 10$, which are the constituent fatty acids of MCT, are strongly involved in the Maillard reaction, resulting in inhibition of the Maillard reaction. Furthermore, the pore distribution after baking was found to be strongly related to AGEs.

\section{Acknowledgments}

This work was supported by the lijima Memorial Foundation for the Promotion of Food Science and Technology to which we are grateful.

\section{References}

1. Seaton TB, Welle SL, Warenko MK, Campbell RG (1986) Thermic effect of medium-chain and long-chain triglycerides in man. Am J Clin Nutri 44: 630-634.

2. Lavau MM, Hashim SA (1978) Effect of medium chain triglyceride on lipogenesis and body fat in the rat. J Nutr 108: 613-620.

3. Gelebter A, Torbay N, Bracco EF, Hashim SA, Van Italie TB (1983) Overfeeding with medium-chain triglyceride diet results in diminished deposition of fat. Am J Clini Nutr 37: 1-4.

4. Chanez M, Bois-Joyeux B, Arnaud MJ, Peret J (1991) Metabolic effects in rats of a diet with a moderate level of medium-chain triglycerides. J Nutr 121: 585594

5. Kris-Etherton PM, Yu S (1997) Individual fatty acid effects on plasma lipids and lipoproteins: human studies. Am J Clin Nutr 65: 1628S-1644S.

6. Kritchevsky D, Tepper SA (1965) Influence of medium-chain triglyceride (MCT) on cholesterol metabolism in rats. J Nutr 86: 67-72.

7. Leveilie GA, Pardini RS, Tillotson JA (1967) Influence of medium-chain triglycerides on lipid metabolism in the rat. Lipids 2: 287-294.

8. Ecelbarger GL, Lasekan JB, Ney DM (1991) In vivo triglyceride secretion and hepatic and plasma lipids in rats fed medium-chain triglycerides, tripelargonin, or corn oil. J Nutr Biochem 2: 260-266.

9. Papamandjaris AA, Macdougall DE, Jones PJ (1998) Medium chain fatty acid metabolism and energy expenditure: obesity treatment implications. Life Sci 62: $1203-1215$

10. Laemmli UK (1970) Cleavage of structural proteins during the assembly of the head of bacteriophage T4. Nature 227: 680-685.

11. Steel R, Torrie J (1980) Principles and procedures of statistics: a biometrical approach (2nd ed). New York: McGraw-Hill.

12. Chi-Hao Wu, Shang-Ming H, Lin J, Gow-Chin $Y$ (2011) Inhibition of advanced glycation endproduct formation by foodstuffs. Food Funct 2: 224-234. 